\title{
VALORES PARA SER MEJORES: LA INVESTIGACIÓN-ACCIÓN EN UNA PROPUESTA VALORAL EN CIUDAD JUÁREZ
}

OLIVERIO ISMAEL FERMAN ÁVILA ${ }^{1}$

\section{RESUMEN}

1 n el artículo se presenta un ejercicio exhaustivo de investigación documental acerca del trabajo que ha desarrollado el Programa Educación en Valores, AC (PEV) por más de veinticuatro años en escuelas de educación básica de Ciudad Juárez, Chihuahua. El trabajo del PEV, se describe a partir del abordaje - de la manera más sintética posible- de trece investigaciones desarrolladas por la propia asociación civil en vinculación con diferentes organizaciones o instancias, tanto del sector público como privado, así como universidades, con el objetivo de determinar variables y/o condiciones que permitan sopesar el impacto de las distintas propuestas de intervención que el Programa ha implementado con la misión de Concientizar a la sociedad (juarense) en la práctica de conductas éticas para lograr una mejor calidad de vida, desde 1994.

Palabras clave: organizaciones de la sociedad civil, educación en valores, investigación, evaluación, medición, impacto.

1 Profesor de Medio Tiempo en la Universidad Autónoma de Ciudad Juárez. México. Correo electrónico: oliverio.ferman@uacj.mx 


\section{INTRODUCCIÓN}

En 1995 nace la asociación civil denominada Programa de Reactivación Social (Progreso) y dentro de esta, el Programa Educación en Valores (PEV), por iniciativa de la comunidad y en respuesta a los problemas sociales, económicos, culturales y urbanos que enfrentaba Ciudad Juárez. Desde sus inicios este programa considera al proceso educativo como base del desarrollo de la sociedad (Ferman, \& Junco, 2006), autodefiniéndose como "una asociación civil sin fines de lucro que pretende coadyuvar en la formación integral del individuo con actividades dirigidas a la práctica de valores éticos en cualquier ámbito" (Ferman, \& Junco, 2006). Esta organización nace con la intención de constituirse como un programa para la familia, pero detonado en la escuela con el firme apoyo y participación de toda la comunidad fronteriza.

El PEV inicia su labor teniendo como misión "garantizar la promoción del cambio permanente de la comunidad juarense hacia mejores actitudes éticas, con la mayor calidad posible..." y visualizándose como "un programa que incida profundamente en los valores éticos de la sociedad local, e impacte como modelo regional y nacional" (Ferman, \& Junco, 2006). Para el desarrollo de su propuesta, el PEV consideró el Modelo de Educación en Valores desarrollado por la licenciada Cecilia Pliego en la ciudad de Saltillo, Coahuila.

En el ciclo escolar 1995-1996, se presentó la propuesta a la Sección 42 del Sindicato Nacional de Trabajadores de la Educación (SNTE). Dicha organización sindical dio su aval para que el pilotaje se iniciara en tres escuelas del nivel básico: un preescolar, una primaria y una secundaria. Así, se integró una comisión de tres profesores que recibió entrenamiento para implantar la propuesta. El plan piloto abarcó cerca de novecientos alumnos, setecientas cincuenta familias y sesenta docentes en tres centros escolares.

Para 1997, se inició el trabajo en treinta escuelas: quince primarias, cinco secundarias y diez preescolares, y es entonces cuando el programa de la licenciada Pliego comenzó a sufrir modificaciones sustanciales para adecuarlo a las necesidades de la ciudad, comen- 
zando por sensibilizar a maestros y padres de familia de retomar la formación valoral con una perspectiva educativa más acorde al constructivismo. El PEV seleccionó dieciocho valores de la propuesta original con el fin de proponer a las escuelas el desarrollo de un valor cada quincena, teniendo como referencia el ciclo escolar que consta generalmente de dieciocho quincenas. De esta manera, se concretó lo que Progreso llamó regionalización del modelo, adoptando oficialmente el PEV. Desde entonces, y hasta la fecha, el PEV ha venido interviniendo en las escuelas de educación básica con distintas propuestas, sin perder la esencia que le dio origen.

Para 2017, el $\mathrm{PEV}^{2}$ reportó la implementación de su proyecto en más de seiscientas escuelas ubicadas en Ciudad Juárez y el valle de Juárez, Chihuahua. A lo largo de todos estos años, a la par de los proyectos de intervención, el PEV ha desarrollado distintos procesos de investigación con el fin de determinar potencialidades y/o debilidades, y en franca búsqueda de variables o condiciones que permitan lograr un mayor impacto en el contexto escolar, así como el cumplimiento de su misión.

Es, por tanto, la intención de este ejercicio de investigación documental describir las variables y/o condiciones que han centrado el interés del programa y que se han constituido en procesos de investigación evaluativa, desarrolladas por distintas instituciones. Se ha considerado importante describir lo más sintético posible cada uno de los procesos de investigación, de tal forma que esto permita tener una visión global de los objetivos y, con ello, de las variables, las metodologías, las muestras y los resultados de cada uno de ellos.

\section{LAS PRIMERAS EXPERIENCIAS DE LA INVESTIGACIÓN}

En 1998 la Coordinación de Investigación y Desarrollo Académico (CIDA) presentó los resultados del estudio denominado "Evaluación del Programa Integral de Valores: resultados y recomendaciones". El diseño de esta investigación fue cuasiexperimental, de tipo

2 Para conocer más acerca de la propuesta actual que implementa el PEv, puede consultar la página oficial de la asociación civil: http://www.educacionyvaloresj.org/ 
comparativo analógico entre escuelas con y sin intervención. La metodología utilizada fue el estudio de caso, teniendo como unidad de análisis a la escuela, pero nutriendo la información a través del levantamiento de datos con los siguientes actores de la comunidad educativa: docentes, directivos, padres de familia y alumnos. Los objetivos más destacables de esta investigación fueron: conocer el desarrollo de la capacitación en cuanto a duración, contenidos, metodología, conducción y participación de los docentes; y conocer la interrelación que existe entre estos y la consecución de los objetivos globales del PEV.

De los resultados descriptivos que presentó el estudio, se destacan los siguientes: todos los docentes encuestados consideraron que la escuela pública debe desarrollar programas de valores; 95 $\%$ de ellos declararon haber trabajado en el tema de los valores desde antes de la implementación del PEv, aunque no de manera sistemática y explícita; los docentes consideraron que $11.9 \%$ de los padres de familia participaron con entusiasmo en las actividades con orientación en la formación de valores y que fueron desarrollados por la escuela; el nivel de agresión en las escuelas sigue existiendo, pero se destacó que en las escuelas piloto predominó la agresividad verbal, mientras que en las testigo resaltó la agresividad física; la escuela control fue percibida por los estudiantes como más autoritaria.

En el ciclo escolar 1999-2000, se firmó por primera vez un convenio de colaboración con la Secretaría de Educación del Estado, lo que permitió a la organización civil alcanzar una cobertura de cien escuelas. Este convenio le abrió al PEv, de manera oficial, las puertas a las escuelas públicas, así como la posibilidad de acceder al presupuesto gubernamental.

Con los resultados antes descritos y el apoyo recibido por el Gobierno del Estado, a partir del ciclo escolar 2000-2001, se presentó la propuesta a la Sección 8 del SNTE, lo que permitió que un mayor número de escuelas participaran en el desarrollo de la propuesta, llegando a un total de ciento sesenta al final de dicho periodo. Con un mayor número de escuelas desarrollando la propuesta, el PEV recurre a la Universidad Autónoma de Ciudad 
Juárez (UACJ) para que realizara una investigación evaluativa que estuvo a cargo del Programa de Trabajo Social, a través de un equipo que desarrolló la investigación denominada "Evaluación del Programa de Educación en Valores-Progreso".

Esta evaluación tuvo como meta amplia convertirse en un estudio de carácter exploratorio como un primer esfuerzo para aproximarse al estado de operacionalización de la propuesta del $\mathrm{PEV}$. La investigación pretendió proporcionar un informe que mostrara los resultados cualitativos y cuantitativos sobre la eficacia, efectividad, validez y confiabilidad de la implementación de dicha propuesta durante el periodo comprendido entre 1996 y 1999.

Para la realización de esta investigación, se determinó una muestra representativa de cuatro instituciones educativas: tres primarias y una secundaria, de un universo de treinta escuelas que desde los inicios del PEv permanecieron implementándolo hasta dicho periodo. El universo de exploración fue, como para la primera investigación, desarrollado por la CIDA y la comunidad escolar en perspectiva amplia: alumnos, padres de familia, maestros y directivos. La UACJ encuestó a 20 maestros acerca de la implementación del programa y de estos, se encontró que únicamente $10 \%$ logró integrar la propuesta del PEV con el currículum oficial, 60 \% lo realizó parcialmente y $30 \%$, muy poco. El $85 \%$ de los docentes refirió haber aplicado el valor quincenal, como se estipuló en el programa. De quienes lograron aplicar el programa, 65 \% manifestó que de la propuesta de 18 valores el más sencillo de adoptar de forma quincenal fue el valor de la amistad (Osio, Pérez, Montoya, Navarro, \& Herrera, 1999). De acuerdo con los resultados de esta evaluación, 95 \% de los maestros afirmó haber aplicado los valores en su relación con las alumnas y alumnos (aspecto subyacente al currículum oculto), y 80 \% de los docentes manifestó la necesidad de la perseverancia en el rescate de los valores dentro de la institución escolar con ayuda de un programa externo como el PEV (Osio et al., 1999).

La UACJ recomendó algunos aspectos de importancia para el diseño y continuidad de la propuesta del PEV en el campo de la educación en valores, precisando tres: la necesidad de definir y 
construir estrategias de acciones comunes entre los actores sociales, que permitan reforzar y aprovechar los recursos y la disponibilidad para el desarrollo de este tipo de programas; luego redefinir algunos aspectos programáticos y operativos del programa en el campo escolar (sin precisar en el reporte de investigación los aspectos a que se refieren específicamente), para que favorezcan la aplicación, desarrollo, continuidad y evaluación del programa; $y$, finalmente, transformar algunas concepciones en materia de normas y valores como reguladores del comportamiento de un individuo dentro de una sociedad en los diferentes espacios de desarrollo (Osio et al., 1999).

Para el ciclo escolar 2001-2002, con trescientas sesenta escuelas implementando la propuesta del PEV y con la visión de crecimiento en la totalidad de escuelas del nivel básico en la localidad, la UACJ vuelve a desarrollar otra investigación en continuidad con la realizada anteriormente. Los resultados fueron presentados en octubre de 2001.

Esta investigación tuvo como objetivo evaluar el impacto de las actividades educativas desarrolladas por el PEV en un periodo de seis meses, para conocer en qué medida y de qué manera se alcanzaron las metas y los objetivos propuestos por la asociación civil; entre estos el cambio de actitudes en maestros en relación con las interacciones con alumnos. La metodología propuesta fue el estudio de varias cohortes, con el objetivo de estudiar prospectivamente dos o más poblaciones de individuos con las mismas características, controlando la temporalidad y la observación de la aparición de un efecto.

Para la realización del estudio, se buscó analizar la relación entre variables de interés, por lo que se consideró: a) Descripción de beneficios potenciales del programa; b) Cálculo y comparación de efectos (actitudes, aprendizaje, cambios de parámetros o proporciones de avance; y c) Patrones de desarrollo y evolución a nivel personal de los sujetos de estudio. En otras palabras, la evaluación intentó describir, de entrada, el impacto de la propuesta del PEV en los usuarios directos: docentes y directivos; y en los indirectos: alumnos (Pérez, \& Puga, 2001). Como usuarios directos se consi- 
deraron los maestros, debido a que la propuesta se orientó a que estos desarrollaran actividades que impactaran en los alumnos.

Se determinaron como rubros centrales de análisis: a) La comprensión de valores y la práctica, tanto en la escuela como fuera de ella (el contexto inmediato: la familia); b) La opinión sobre la clase de valores y una percepción sobre el material de apoyo; el nivel de compromiso de la comunidad escolar asumido con el $\mathrm{PEV}$, tomando como base el cumplimiento de la propuesta (valores quincenales a lo largo del ciclo escolar: un valor desarrollado cada quincena); y c) Tareas desarrolladas por las escuelas y manifestación de interés por parte de la población escolar, así como beneficios del programa en las relaciones a nivel interpersonal, grupal (colectivo pedagógico), familiar y personal.

Entre los resultados más significativos, se destaca que se observó interés en alumnos, padres de familia y maestros para integrar la práctica de valores en su vida cotidiana, quienes opinaron que las actividades en torno al tema de los valores resultan novedosas en el contexto escolar. Los investigadores concluyeron que "sin duda alguna, el proceso de enseñanza en materia de Educación en Valores llevada a cabo brinda la oportunidad a cada uno de los alumnos, docentes, padres de familia y otros de construir una nueva cultura escolar" (Pérez, \& Puga, 2001). Según el propio estudio, esta nueva cultura va de la mano con la buena intención que conlleva el PEv, pues su carácter globalizante e integrador en la práctica pedagógica, crea y recrea un ámbito (valores) y las condiciones necesarias para desarrollar no solo la unidad del enseñar y aprender (Ander-Egg, citado por Pérez, \& Puga, 2001), sino también para superar las disociaciones que suelen darse entre la teoría y la práctica, la educación y la vida, así como los procesos intelectuales, volitivos y afectivos; y esto requiere de una metodología que potencie el desarrollo equilibrado de estos aspectos desde una perspectiva epistemológica, pedagógica y didáctica.

En ese momento el programa de valores consistía en la aplicación quincenal de un valor, la utilización de frases y un periódico mural alusivo al valor, así como charlas con alumnos y padres de familia. Los investigadores de la UACJ determinaron que la pro- 
puesta del PEV contenía un enfoque holístico y de carácter globalizador; sin embargo, el doctor Pablo Latapí Sarre (2003) evaluó en ese mismo periodo el enfoque teórico-práctico de la propuesta del PEV y la definió con un enfoque prescriptivo-exhortativo y con una orientación valoral de tipo "empresarial y de formación del carácter” (Latapí, 2003, p. 154), misma valoración que le otorgó al Modelo de Enseñanza Activa de Valores Éticos (EAVE), implementado en Coahuila en 1997, de donde parte la propuesta del PEV (Latapí, 2003, p. 154).

\section{UNA NUEVA PROPUESTA A PARTIR DE LAS PRIMERAS EXPERIENCIAS DE INVESTIGACIÓN}

En el ciclo escolar 2001-2002, la influencia del PEv llegó a trescientas sesenta escuelas, pero para el siguiente ciclo escolar la cobertura alcanzó quinientas cincuenta en ambos subsistemas (federalizado y estatal). En este último periodo, la metodología es transformada: se deja de lado la planeación quincenal; pasa de concebirse como un programa prescriptivo para ser precisado como propositivo con un enfoque vivencial (Ferman, \& Junco, 2006).

La propuesta de dieciocho valores quincenales, se convierte en un marco de referencia para la institución educativa y no en una norma establecida, ya que en concordancia con un diagnóstico de la realidad institucional es la propia escuela la que determina la necesidad de desarrollo y/o aprehensión de determinados valores en la comunidad escolar. Se propuso el diseño y puesta en práctica de los valores, a través de planeaciones colectivas, sin ajustarse a un tiempo específico, donde no solo el colectivo pedagógico participara, sino toda la comunidad escolar. Para consolidar una nueva propuesta, el PEV modificó los sustentos epistemológicos y metodológicos, y consideró cinco orientaciones que, a partir de ese momento, lo caracterizarían: programa preventivo y no correctivo; propositivo, no prescriptivo; dirigido a los adultos educadores; programa centrado en el desarrollo de la persona; y búsqueda de armonía en el desarrollo de la esfera intelectual, 
afectiva y actitudinal, a partir de los postulados de teorías psicopedagógicas contemporáneas.

Se propone, entonces, que el sustento filosófico se convierta en lo que el PEV denominó como camino para la aprehensión de los valores. Teniendo como basamento las cuatro virtudes morales cardinales: justicia, fortaleza, autogobierno y prudencia (que se desprenden de los cuatro valores fundantes en la propuesta del Programa EAVE). Cada una de ellas como peldaño en orden ascendente. La justicia toma sentido como el proceso de enamoramiento del valor: "Tener claro el bien que se desea alcanzar"; la fortaleza, como segundo peldaño, se constituye en el primer paso para la vivencia propiamente del valor; se define, pues, como "alcanzar el bien deseado” (Ferman, \& Junco, 2006). El tercer peldaño, autogobierno, se centra en "Mantenerse en el bien" a través de actividades planeadas por el colectivo con ese fin; y, finalmente, el último escaño, la prudencia, desarrollada esta virtud mediante actividades que permitan a la persona "distinguir cuándo, dónde y cómo hacer uso de ese bien”. Este camino de la aprehensión parte de un método desarrollador de tipo experiencial, que se inicia necesariamente de la práctica (vivencia) inducida a través de la esfera afectiva (de los sentimientos), que propicia un proceso de reflexión y, finalmente, permite a la persona la construcción de la experiencia (teorización) (Ferman, \& Junco, 2006).

Para finales del ciclo escolar 2002-2003 e inicio del siguiente, la empresa de consultoría Bismark realizó una evaluación sobre el impacto del PEV. La muestra fue de 492 maestros y 164 directivos. Esta evaluación se desarrolló desde la perspectiva del conocimiento, involucramiento, implementación e impacto, y con el objetivo general de determinar el impacto del Programa Educación en Valores en los diferentes actores de este, estableciendo los diferentes valores entre personas expuestas al programa y personas no expuestas al mismo.

Es importante mencionar que esta evaluación se realizó cuando la incorporación de la nueva propuesta del PEV había llegado a la gran mayoría de las escuelas. Dentro de esta nueva propuesta del PEV, se establecieron dos resultados (impactos) deseados: 1) 
Formar niños y adolescentes que no solo reciban herramientas cognoscitivas o laborales, sino formación humana, social, cívica, fundamentada en valores, creencias y actitudes sociales adecuados para la convivencia y la calidad de vida de toda la sociedad juarense; y 2) Modificar o reforzar — según sea el caso- creencias y actitudes de los educadores y de los padres de familia, quienes llevan a cabo el proceso de educación de manera más directa e inciden en lo que niños y adolescentes serán en el futuro. Esta parte es esencial, ya que es necesario concientizar a estos actores de su grado de responsabilidad y de la importancia de emitir mensajes éticos a las nuevas generaciones.

Los resultados más significativos con respecto a los docentes fueron (Bismark, 2004): un porcentaje alto no considera la formación en valores como urgente, ya que le adjudican el tercer lugar en importancia después de los conocimientos y habilidades; respeto y responsabilidad son los valores fundamentales; sin embargo, se observó que el maestro da al respeto una connotación de subordinación y obediencia que no coincide con la nueva propuesta educativa del PEV; 20 \% de los maestros que se capacita con las nuevas orientaciones no lo pone en práctica; la capacitación es insuficiente y no todos los maestros la toman, lo que hace que el programa se desarrolle bien en algunos ciclos y en otros no, disminuyendo o anulando, en su caso, la eficiencia en cuanto a impacto; $44 \%$ opina que la escuela y los papás deben encargarse de la enseñanza de valores; $34 \%$, que la escuela, la Iglesia y los papás; el programa sí muestra haber tenido impactos significativos en hasta $6 \%$ en los niños.

Se destacan dos observaciones presentadas por Bismark: a) Es un programa de largo plazo, el cual irá rindiendo frutos paulatinamente, y tres generaciones completas de llevar de manera sistemática el programa año tras año; y b) Tanto los directivos como las instituciones que lo apoyan (al PEv), deben considerar que gran parte de las variables incidentes en el niño para la aprehensión del mensaje, se encuentran fuera del ámbito del programa y que, incluso, aquellas dentro de sus dominios se escapan en el momento mismo en el que el maestro se encuentra frente al grupo. 


\section{LOS VALORES Y LAS CONDUCTAS DE RIESGO}

Entre 2003 y 2004, la Fundación del Empresariado Chihuahuense (Fechac), benefactora del PEV, propone la realización de una evaluación de impacto en los jóvenes de secundaria y alumnos de primaria mediante un estudio cuasi-experimental en escuelas que desarrollan el PEV (grupo experimental), y considerando en el grupo control a escuelas que no desarrollan la propuesta del PEV, con el fin de contrastar los resultados obtenidos en cada uno de los grupos. Este estudio se denominó "Evaluación Programa Educación en Valores" y fue liderado por el doctor Enrique Suárez y Toriello (2005) y los resultados presentados en marzo de 2005.

El objetivo del estudio fue generar información que pudiera: a) Sustentar la importancia del PEV y b) Demostrar el impacto de la intervención del programa en lo que respecta a la formación de capital humano y en la reducción de conductas de riesgo que afectan el desarrollo saludable de niños y jóvenes. La muestra estuvo conformada por 445 estudiantes de 14 escuelas: 9 primarias y 6 secundarias. La selección de la muestra consideró el mismo número de planteles en el grupo control, así como escuelas que recién comenzaban con el programa (nivel II) y aquellas que tenían un grado avanzado de desarrollo del PEV (nivel I).

Se evaluaron 11 conductas de riesgo que — se consideró- tienen un impacto negativo en el crecimiento y desarrollo de niños y jóvenes. De ellas, 4 se relacionaron fundamentalmente con el ambiente donde se desarrolla el niño/joven y 7 refieren a acciones que son llevadas a cabo por el niño/joven. En promedio, los entrevistados tuvieron 3.53 conductas de riesgo con una desviación estándar de 2.02 conductas riesgosas en un rango de 0 a 10 conductas de riesgo, donde 0 representa la ausencia.

Los resultados se consideraron alentadores y entre los más significativos están: los alumnos del grupo experimental presentaron una menor prevalencia de conductas violentas que los del grupo control en -10 \%; tabaquismo, -5\%; uso de alcohol, -6 \%; y uso de drogas, -5 \% (Suárez y Toriello, 2005). Así como una mayor predisposición a la práctica de valores. 
Gráfica 1. Porcentaje de jóvenes que usaron algún tipo de droga en el último año, según el nivel de intervención

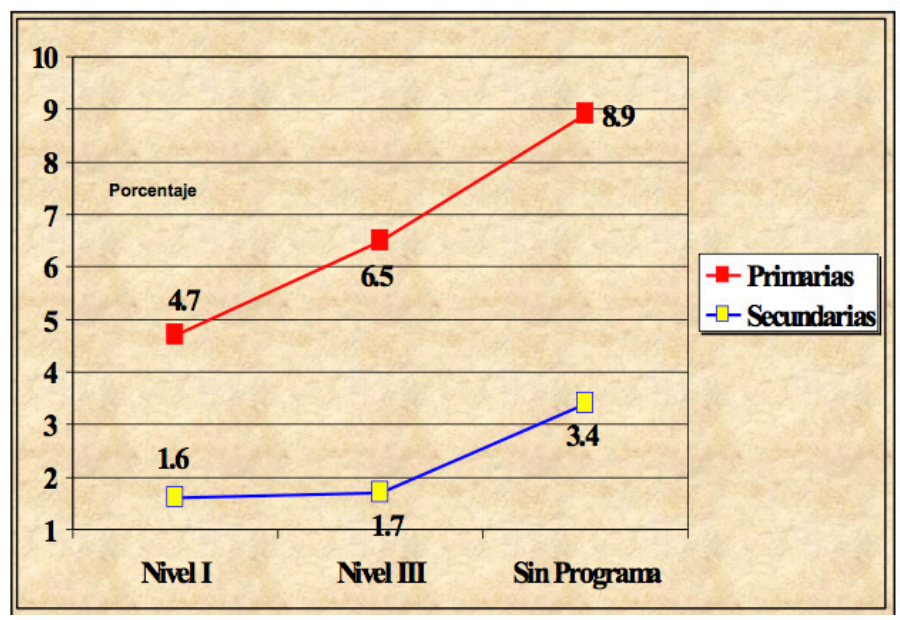

Fuente: Suárez Toriello, 2005.

De los resultados obtenidos en el estudio, se desprendieron las siguientes recomendaciones (Suárez, 2005): continuar desplegando la iniciativa con carácter de prioritario, con el objetivo de gestar el cambio social que mejore niveles de bienestar y la calidad de vida de la población; intensificar la participación activa de los padres de familia y de la sociedad en su conjunto en el desarrollo de actividades de valores, a fin de que el impacto deseado de esta iniciativa sea más significativo; y que se desarrollen estrategias de intervención que promuevan y faciliten la práctica cotidiana de los valores como principal instrumento que permita la internalización y apropiación de los conceptos implícitos en cada uno de los valores.

En el ciclo escolar 2005-2006, se desarrolla otra evaluación liderada por el doctor Enrique Suárez y Toriello (2005) con los mismos objetivos que la anterior. Cabe destacar que esta evaluación fue más exhaustiva que la primera, teniendo como mayor cuidado la selección de la muestra que estuvo conformada por ochocientos estudiantes de catorce escuelas primarias y veintidós secundarias ubicadas en trece zonas geográficas que fueron previamente 
definidas por el PEV. De estos, $75 \%$ acudía al turno matutino y $25 \%$ al vespertino. La selección de las escuelas participantes consideró el mismo número de planteles que no habían desarrollado un programa de valores (grupo control), así como de escuelas más comprometidas con el desarrollo del programa (nivel i/ grupo intervención). Cada muestra tuvo la misma representación numérica y proporcional. Una vez aplicados los cuestionarios, la información recabada fue capturada y analizada usando el Programa Estadístico para las Ciencias Sociales (sPss, por sus siglas en inglés). El análisis de los grupos de control y de intervención no mostró diferencias significativas en lo que respecta a edad, sexo, escolaridad y condición socioeconómica, implicando la similitud de ambos grupos para efectos de las comparaciones que el estudio quiso realizar, a fin de demostrar la importancia del PEV en la disminución de las conductas de riesgo en la población estudiada. Cada uno de los dieciocho valores fue evaluado a través de tres preguntas específicas y se estableció, para propósitos del análisis, que el entrevistado practicaba el valor si después de promediar los registros numéricos de las tres respuestas alcanzaban $75 \%$ de respuestas favorables. La misma norma se empleó para evaluar la presencia o no de conductas de riesgo, considerando que estaba presente si tenían $75 \%$ de las respuestas a favor. El estudio determinó, tal como en la evaluación anterior, un total de once conductas de riesgo. 
Tabla 1. Jóvenes que presentan conductas de riesgo

\begin{tabular}{cc}
\hline Conducta de riesgo & $\%$ \\
\hline Falta de límites (casa, escuela) & 28.3 \\
\hline Ausentismo escolar voluntario & 30.6 \\
\hline Uso inadecuado del tiempo libre & 83 \\
\hline Conducta violenta (violencia familiar, pleitos físicos) & 35.4 \\
\hline Depresión & 54.4 \\
\hline bebidas alcohólicas seguidas una o más veces en: U/6m; U/M & 19.9 \\
\hline Cigarrillos fumados en: AvV; U/A; U/M & 20.5 \\
\hline Múmero de veces de uso de drogas: AvV; U/A; U/M & 8.6 \\
\hline Conducta antisocial (robo en casa y/o en tienda) & 25.8 \\
\hline Ambiente familiar negativo & 21.6 \\
\hline
\end{tabular}

Fuente: elaboración propia, a partir de Suárez Toriello, 2005.

La tabla 1 muestra la frecuencia general de las conductas de riesgo analizadas en los alumnos de las escuelas primarias y secundarias. En ella, se observa que $28 \%$ se desarrolla en un ambiente caracterizado por la falta de límites impuestos por la familia y/o por la escuela. Tres de cada diez alumnos incurrieron en el ausentismo escolar voluntario en el mes previo a la aplicación de la encuesta. El 83 \% hace un uso inadecuado de su tiempo libre, es decir, no aprovecha constructivamente el tiempo una vez que sale de la escuela; 35 \% manifestó estar expuesto a violencia doméstica (24\% fue golpeado por sus padres tres veces o más en el último mes) y/o haber desarrollado una conducta violenta (24 \% se peleó tres o más veces en el último mes). Un poco más de la mitad de los alumnos manifestó haber tenido depresión en los tres meses previos a la aplicación de la encuesta (38 \% experimentó depresión en el último mes).

Dos de cada diez jóvenes ingirieron durante una o más ocasiones cuatro o más bebidas alcohólicas seguidas en los últimos seis meses (11 \% en el último mes); 21 \% de los jóvenes ya ha fumado cigarrillos (8 \% ha fumado cinco o más cigarrillos por día en el último mes); 9 \% ha usado drogas ilegales (8 \% empleó drogas ilegales una o más veces en el último mes); $26 \%$ de los niños y 
jóvenes está expuesto a malas compañías (21 \% tiene amigos que utilizan drogas; 27 \% tiene amigos que toman bebidas alcohólicas una o más veces por semana y $28 \%$ tiene amigos que se meten constantemente en problemas); $22 \%$ manifestó algún tipo de conducta antisocial (28 \% robó dinero de sus padres en el último año y $14 \%$ se robó algo de una tienda en el último año).

Más de la mitad de los alumnos entrevistados presentó un ambiente familiar negativo (66 \% no tiene comunicación con sus padres y $21 \%$ vive en un ambiente en donde sus padres se gritan y pelean de manera regular). Para evaluar el impacto del PEV en las conductas de riesgo, se comparó a los dos grupos referidos (intervención y control) de acuerdo con el número de valores, es decir, jóvenes con nueve valores o menos y adolescentes con diez a dieciocho valores. Se consideró en el análisis que el verdadero impacto del programa, se puede percibir en los jóvenes con diez valores y más (véase tabla 2).

Tabla 2. Conductas de riesgo en jóvenes

\begin{tabular}{ccccc}
\hline $\begin{array}{c}\text { Conductas de riesgo } \\
\text { Jóvenes con diez valores y } \\
\text { más }\end{array}$ & $\begin{array}{c}\text { Intervención } \\
\text { (nivel I) }\end{array}$ & Control & $\begin{array}{c}\text { Diferencia } \\
\text { a favor del } \\
\text { PEV (\%) }\end{array}$ & $\begin{array}{c}\text { Relación } \\
\text { Control-PEV }\end{array}$ \\
\hline $\begin{array}{c}\text { Ausencia de límites } \\
\text { Ausentismo escolar volun- } \\
\text { tario }\end{array}$ & 16.8 & 23.4 & -6.6 & 1.39 a 1 \\
\hline $\begin{array}{c}\text { Uso inadecuado del tiempo } \\
\text { libre }\end{array}$ & 23.7 & 31.7 & -8 & 1.34 a 1 \\
\hline $\begin{array}{c}\text { Conducta violenta } \\
\text { Depresión }\end{array}$ & 75.3 & 83.9 & -8.6 & 1.11 a 1 \\
\hline Abuso del alcohol & 50.2 & 37.3 & -10.1 & 1.37 a 1 \\
\hline Tabaquismo & 12.2 & 18.1 & -2.6 & 1.05 a 1 \\
\hline Uso de drogas & 13.6 & 18.7 & -5.1 & 1.48 a 1 \\
\hline Malas compañías & 5.9 & 9.9 & -4 & 1.68 a 1 \\
\hline Conducta antisocial & 15.4 & 29.0 & -13.6 & 1.88 a 1 \\
\hline Ambiente familiar negativo & 8.2 & 20.6 & -12.4 & 2.51 a 1 \\
\hline
\end{tabular}

Fuente: elaboración propia, a partir de Suárez Toriello, 2005. 
En la tabla 2 se presentan los porcentajes de jóvenes en las diversas conductas de riesgo determinadas en el marco del estudio, y estas según su participación en el PEV y la relación con el número de valores que tienen (sic) (diez y más valores). Las diferencias que presentan los resultados en la tabla, con excepción de depresión y ambiente familiar negativo, son estadísticamente significativas, debido a que los autores consideran que la diferencia es atribuible a la intervención del PEV. La significancia estadística se estableció con base en la prueba de Chi cuadrada de Pearson, con un grado de confianza de $95 \%$ o mayor. Cabe destacar que en la última columna, se presenta el número de jóvenes del grupo control con la referida conducta por cada adolescente que tuvo la experiencia de participar en el PEV. De acuerdo con estos resultados, se puede hacer notar que, por ejemplo, por cada joven que participó en el PEV hay 2.5 adolescentes del grupo control que tienen conducta antisocial, 1.88 jóvenes con malas compañías, 1.68 adolescentes que usan drogas, 1.48 jóvenes que abusan del alcohol y 1.38 adolescentes que fuman tabaco de manera regular.

Debido a que los resultados del estudio antes descrito, se publicaron en la prensa, un estudiante del Doctorado en Educación de la Universidad de Texas en El Paso (uteP), René Rubio Pallares, se acercó al PEV con el fin de conocer a detalle las últimas experiencias de investigación desarrolladas por el doctor Enrique Suárez Toriello (2005). Como parte de su trabajo de tesis doctoral, Rubio Pallares solicitó a la Dirección Ejecutiva del PEV poder analizar, en primera instancia, el instrumento que sirvió de base para la obtención de los datos que dieron origen a los resultados presentados en las evaluaciones. Con la anuencia del PEV, Rubio Pallares modificó la herramienta utilizada para afianzarla como instrumento estandarizado de alta confiabilidad y que facilitara la medición de la práctica de valores. Se denominó a este proyecto "The construction of attitudinal scales to appraise valuesembedded education", ${ }^{3}$ que fue asesorado por el doctor Rodolfo Rincones.

3 La construcción de una escala actitudinal que valore los valores implícitos en la educación. 
Después de una primera aplicación de la nueva herramienta en diez escuelas que desarrollaban la propuesta del PEV y diez planteles que no, se presentó un informe que a la par enunciara el proceso de pilotaje de los instrumentos, en donde se destacan algunos resultados de las mediciones: el juicio de valoración sobre el funcionamiento general de los instrumentos para la medición de valores presenta una alta confiabilidad; se consideran apropiados para la medición futura de este tipo de predisposición actitudinal de los alumnos; se sugieren múltiples aplicaciones en el corto plazo con el fin de realizar pruebas que certifiquen los niveles de validez y confiabilidad en distintos escenarios de la ciudad en donde se desarrolla la propuesta del PEV; y, finalmente, los datos obtenidos en esta primera aplicación no sugieren que el programa sea un factor en la respuesta de los alumnos. El investigador concluyó que, por tanto, el programa podría sustentar su efectividad, ya que en el comparativo entre estudiantes inscritos en escuelas que desarrollaban el programa con aquellos que no recibieron influencia del PEV, no se encontró ninguna diferencia significativa.

\section{LAS IDEOLOGÍAS EDUCATIVAS EN LA PRÁCTICA DE VALORES}

Con los recientes resultados de la investigación que pusieron en duda el impacto del programa, la dirección ejecutiva de la asociación civil se dio a la tarea de buscar investigadores que orientaran sus inquietudes en propuestas en otro sentido, considerando en primera instancia que la capacitación para la implementación de la propuesta, se dirige a los maestros. De las diversas propuestas analizadas, el PEV optó por la presentada por el licenciado Roberto Anaya Rodríguez, candidato a maestro en Ciencias Sociales por la UACJ. La investigación que realizó Anaya (2007) se tituló "Razonamiento moral, ideología educativa y programas de valores", cuyo diseño fue no experimental-transeccional descriptivo y comparativo. Dicha investigación estableció dos variables: grado de desarrollo del razonamiento moral en alumnos y maestros; y la ideología educativa como el elemento que orienta la práctica docente: estilo de enseñanza y liderazgo. 
Esta investigación se convirtió en una oportunidad de realizar un análisis de la ideología educativa, tanto en maestros que desarrollan la propuesta del PEV como en otros que no, efectuar un comparativo y poder determinar el inventario de ideologías educativas presente en los docentes de escuelas que desarrollan la propuesta del PEV. El análisis de las ideologías educativas, se consideró importante toda vez que esta se considera como

El conjunto de opiniones, actitudes y valores que acerca de la educación, tienen los maestros; la perspectiva acerca del propósito de la educación, sus objetivos secundarios, el papel del alumno en el proceso de enseñanza aprendizaje, la selección del conocimiento válido, la importancia del control disciplinario, la función social y profesional de profesor, los valores deseables a promover, la escuela como organización institucional y el papel que debe jugar la comunidades en general, los padres de familia y el Estado en particular en el diseño curricular de las experiencias educativas (Arroyo, 1998, p. 67).

En los resultados acerca de la ideología educativa presente en los maestros, la investigación destacó lo siguiente:

Tabla 3. Medias por rasgos de ideología educativa para escuelas públicas con y sin programa de valores

\begin{tabular}{cccc}
\hline Tipos de ideología & General & Con programa & Sin programa \\
\hline Fundamentalismo & -2.71 & -3.48 & -1.56 \\
\hline Intelectualismo & 3.27 & 3.78 & 2.50 \\
\hline Conservadurismo & 7.98 & 7.04 & 9.39 \\
\hline Liberalismo & 9.78 & 9.74 & 9.83 \\
\hline Liberacionismo & 7.40 & 7.48 & 7.28 \\
\hline Anarquismo & 0.04 & 0.85 & -1.17 \\
\hline Conservador general & 1.76 & 1.00 & 2.89 \\
\hline Liberal general & 8.16 & 8.07 & 8.28 \\
\hline Total de casos & 45 & 27 & 18 \\
\hline
\end{tabular}

Fuente: Anaya, 2007. 
VALORES PARA SER MEJORES: LA INVESTIGACIÓN-ACCIÓN EN UNA PROPUESTA...

Como destaca Anaya (2007), en el estudio

resulta interesante, que a pesar de que las diferencias entre los perfiles no son significativas, para el caso de las escuelas con programas de valores en donde igualmente el tipo de ideología que califica más alto es el liberacionismo, en este caso el tipo de ideología que le sigue es el liberacionismo y no el conservadurismo como es el caso de las escuelas sin programa de valore, en donde el conservadurismo puntúa más alto en dos puntos que el liberacionismo (p. 8).

Resulta interesante analizar el tipo de ideología presente, o bien, preponderante en los docentes que desarrollan la propuesta del PEV y visualizar, con ello, la resistencia y la concordancia con el tipo de actividades didácticas enfocadas en el desarrollo de valores y/o actitudes.

Con los resultados de esta evaluación, la coordinación del PEV se dio a la tarea de realizar una investigación más exhaustiva sobre las ideologías educativas, relacionar esta variable con otras y poder determinar algunas estrategias del PEV. Este trabajo de investigación se desarrolló como parte de una tesis de maestría bajo la dirección de la Universidad Virtual del ITESM. ${ }^{4}$ En la muestra se consideró a maestros de escuelas primarias en Ciudad Juárez, Chihuahua, durante el ciclo escolar 2007-2008, y fue presentado ante la comunidad educativa de Ciudad Juárez en febrero de 2009.

La principal orientación fue indagar acerca de los campos ideológicos de los maestros y su correspondencia con las técnicas didácticas para la enseñanza de valores en el contexto escolar. Como marco de referencia, se tomó la experiencia que ha desarrollado el PEV y con este, poder determinar si las propuestas actuales para la enseñanza de valores están posibilitando la selección de técnicas acordes a los campos ideológicos que los especialistas consideran como necesarios para desarrollar adecuadamente valores y actitudes.

4 La tesis completa puede consultarse en https://repositorio.itesm.mx/ortec/bitstream/11285/568696/1/DocsTec_6458.pdf 
El enfoque desarrollado fue mixto con un diseño de investigación de dos etapas cuasi-experimental (grupo experimentalcontrol). La muestra se determinó no probabilística, docentes de seis escuelas para el grupo experimental (PEV) y seis para el grupo control. La técnica de recolección de datos fue el sondeo y para la recolección de datos, se utilizó: 1) Instrumento estandarizado de ideología educativa (Inventario de Ideologías Educativas, original de O’Neill [1981] y adaptación de Arroyo Picard [1998]); 2) Análisis de contenido (planeaciones de valores); y 3) Entrevista estructurada (a directores). La validez de la información, se determinó por un análisis factorial (Ferman, 2007).

Dentro de los resultados más significativos presentados en esta investigación están: la diferencia más significativa puede establecerse, entre ambos grupos, en la ideología fundamentalista: mientras que el grupo experimental (escuelas PEv) mantienen una predisposición negativa, el grupo control muestra una tendencia positiva, lo que permite sentar una senda diferencia entre los grupos de maestros. La ideología liberalista, que privilegia la función social de la escuela, prevalece en ambos casos, con una ligera ventaja para los docentes en el grupo control. Sin embargo, y pese a las diferencias evidentes en algunas medias como en la ideología fundamentalista entre el grupo experimental (con alejamiento de esta) y el grupo control (con acercamiento), se determinó necesario realizar la prueba estadística denominada $t$. Esta es una prueba estadística que ayuda a evaluar si dos grupos difieren significativamente con respecto a sus medias (Hernández, 2006). 
Gráfica 2. Contraste de ideologías educativas entre escuelas donde se intervino con el PEV versus aquellas donde no se realizó alguna intervención

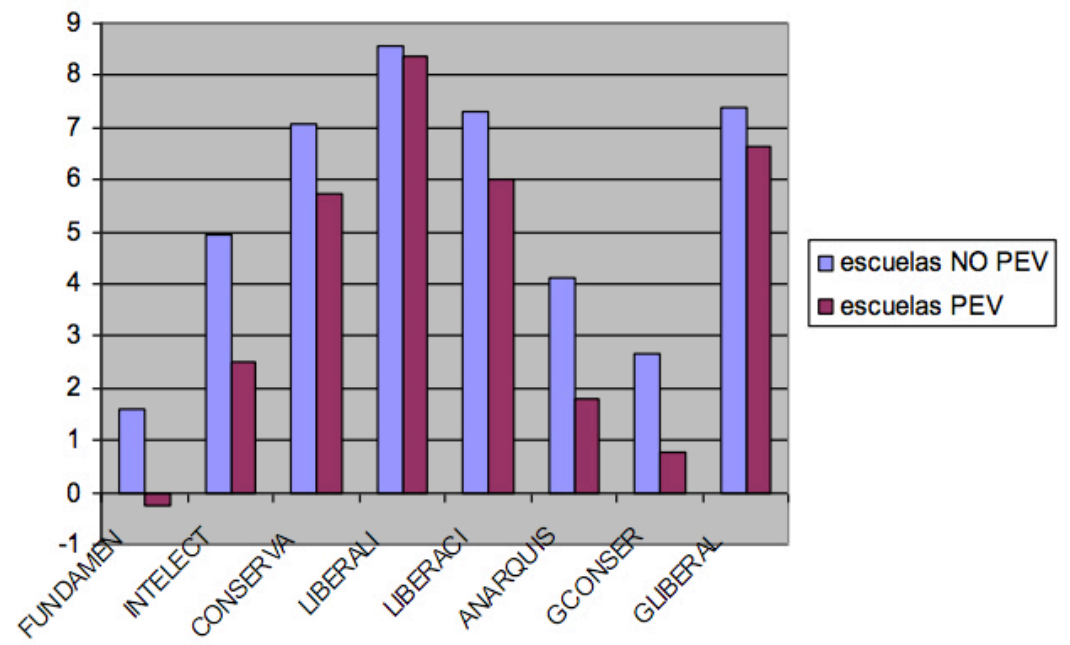

Fuente: Ferman, 2007.

En los resultados de la prueba estadística t, se contrastó el nivel de significancia con respecto al valor de $t$ obtenido en cada variable (campos ideológicos) en cada uno de los grupos (experimental y control), arrojando como resultados en todos los casos que la diferencia entre los resultados entre un grupo y otro es mayor a 0.05, es decir, con probabilidad de error mayor a $5 \%$, por lo que no se establece una diferencia estadísticamente significativa. Adicionalmente, se realizó un análisis de varianza unidireccional (Anova oneway) con el fin de corroborar o descartar la ausencia de diferencias significativas entre las medias obtenidas en los maestros del grupo experimental (escuelas PEv) y el grupo control (escuelas No-PEv), cuyos resultados corroboran que no existe una diferencia significativa entre las medias y las varianzas entre los grupos.

Además del análisis cuantitativo, se analizaron doce planeaciones de actividades para el desarrollo de valores contenidas en los proyectos de cada escuela, seis de planteles que desarrollaban la propuesta del PEV y seis que no participaron en esta (No-PEV), o bien, escuelas que — se consideró- dejaron de aplicar la propues- 
ta en el ciclo escolar 2006-2007 y que, por tanto, implementaron una propuesta propia.

En las planeaciones se analizaron las particularidades de los enfoques de enseñanza de valores en el contexto escolar, según los planteamientos de Pablo Latapí (2003). De los resultados, se destaca que tanto las escuelas que desarrollan la propuesta del PEV como las que no, presentan en su mayoría actividades que privilegian el enfoque prescriptivo, ya que las actividades para la enseñanza de valores se acotan a exposiciones, pláticas, imposiciones por parte de los adultos de valores consensuados solo por ellos, reglamentos, uso de premios y sanciones, entre otras. Solo una de las seis planeaciones de las escuelas PEV presenta actividades con enfoque clarificativo (uso del debate para el establecimiento de reglas dentro del salón de clases, lectura del reglamento escolar, discusión de sanciones por el quebranto de reglas) en tanto que tres de seis planteles No-PEv (50 \%) plantean actividades de este tipo.

Dos escuelas en cada grupo plantean actividades que privilegian el enfoque reflexivo (reflexión de la necesidad de plantar árboles después de ver un video sobre el tema, por ejemplo), aunque en este caso las actividades parten de acciones muy básicas (lecturas, escuchar un audio, paseo por la escuela o la comunidad) que no explicitan las formas en las que serán abordadas con los alumnos; en algunos casos ni siquiera se anotan los datos del material audio o visual a utilizar, dejando demasiado amplio el espectro cuando se anota Lectura sobre el tema, por citar un ejemplo.

A los directores se les indagó sobre el tiempo en el que sus escuelas han participado desarrollando la propuesta del PEV y se obtuvo una media de 2.8 años; algunos de los directores argumentaron desconocer el tiempo que la escuela tiene participando y otro solo unos meses; por tanto, estos planteles se consideraron como 0 . La escuela con mayor tiempo desarrollando la propuesta fue de siete años. Cabe mencionar que un director mencionó que ningún año han desarrollado formalmente la propuesta. Cuando se indagó con los directores sobre el tipo de actividades, estrategias y/o técnicas que desarrolla su plantel con el apoyo del PEV, una respuesta constante fue: frases en honores a la bandera; periódicos murales; 
carteles; desarrollo de trabajo de valores por semana o por quincena; pláticas con alumnos y padres de familia. Al indagar con los directores acerca del impacto de las actividades antes descritas, quienes consideran que favorecen el desarrollo de valores en el contexto escolar, las respuestas oscilaron desde un "educar con el ejemplo" (postura ideológica conservadora) hasta un "positivamente, porque los niños son más conscientes de su cuidado y respeto a su salud y persona". Obviamente si los directores consideran que el impacto ha sido positivo, se tiene que mencionar que este corresponde a una propuesta que el programa ya no considera, pero que, sin duda, puede reflejarse en las respuestas porque se sigue desarrollando en el contexto escolar.

Al preguntarles a los directores si consideran que el PEV es un apoyo para la formación de valores, todos coincidieron en que sí: unos mencionaron el aspecto de la sensibilización de la tarea formativa en el contexto escolar; uno considera que el programa ayuda a contrarrestar los efectos negativos de los medios de comunicación y el ambiente; otro considera que es necesario, "porque faltan muchos valores en la casa y la escuela se convierte en el segundo hogar"; y otro considera que el PEV "ayuda a adquirir valores y ponerlos en práctica”. Los resultados obtenidos en la encuesta a directores de escuelas que desarrollan la propuesta del PEV, se corresponden plenamente con los resultados encontrados en las planeaciones de valores analizadas y se concluye que la nueva propuesta del PEV no ha logrado impactar en la práctica docente. A pesar de que el paradigma constructivista es el marco de acción para la tarea docente, pareciera que en la cuestión de desarrollo de valores la práctica está muy compenetrada de la ideología conservadora: se sigue considerando al maestro como un ejemplo moral; aun y cuando teóricos como Latapí (2003) y Schmelkes consideran otros aspectos importantes para el desarrollo de valores dentro de la escuela, entre ellos, las actividades consideradas para que los alumnos experimenten la práctica de valores.

Finalmente - a partir de los resultados presentados en esta investigación-, se destaca que los resultados permiten determinar que las ideologías educativas precisan, en gran medida, la selec- 
ción de estrategias y/o técnicas para la enseñanza de valores en el contexto escolar y que es necesario que las propuestas para el desarrollo de valores en el contexto escolar establezcan sus posturas ideológicas en correspondencia con los enfoques de la enseñanza de valores, para que se puedan establecer estrategias de acción que fortalezcan las propuestas y, de esta manera, se logre romper paradigmas y estereotipos.

\section{TRABAJANDO EN LA CONSTRUCCIÓN DEL CLIMA MORAL}

A partir de los resultados de las investigaciones propias, el PEV implementa desde el ciclo escolar 2008-2009 lo que denominó Proyecto de Valores Éticos en la Escuela (Provee) orientado a la construcción de un mejor clima moral en los planteles. Desde la perspectiva planteada por el PEV, el clima moral parte de enfoques educativos que conciben a la escuela como el lugar por excelencia donde se aprende a convivir y se construyen socialmente los valores. Esta construcción debe darse al interior de la institución escolar a través del planteamiento de estrategias didácticas adecuadas y en un clima propicio que permita el ejercicio de la reflexión sistemática y la toma de decisiones.

Distintas propuestas en el plano internacional consideran la importancia de construir un clima que posibilite, a su vez, la puesta de la aprehensión y la puesta en práctica de los valores. Por mencionar, el Programa de Educación en Valores (Peva), una iniciativa del Instituto de Ciencias de la Educación de la Universidad de Barcelona, sostiene que existen, al menos, dos vías en los procesos de construcción de valores en las instituciones escolares: la primera, orientada a la construcción de valores a través del desarrollo transversal del currículo por medio de estrategias didácticas; y la segunda, la construcción de valores y actitudes mediante el propio proyecto de gestión que busque un buen clima moral. Según esta propuesta, ambas vías son imperativas, interrelacionadas y alimentadas entre sí. Y es precisamente en esta última donde se habla de la construcción de un clima escolar propicio para el desarrollo de esas actitudes y valores. 
Partiendo de esta segunda premisa, el PEV desarrolló una propuesta en las escuelas de educación básica de Ciudad Juárez, Chihuahua (Provee) buscando impactar en la formación de actitudes y/o valores a corto, mediano y largo plazos. El Provee parte de la hipótesis de que toda institución escolar debe avalar su función social con un proyecto educativo (PE) que exprese el ideal de calidad educativa al que se compromete y con el cual se responsabiliza ante la comunidad. Dicho proyecto debe ser la conjunción de lo académico (parte informativa) y lo ético (parte formativa). La propuesta del PEV se orientó en asesorar al colectivo escolar en la elaboración de un proyecto que permitiera a la comunidad educativa sustentar el éxito académico, a partir del afianzamiento de sus valores éticos y a mediano plazo expandir su influencia en la comunidad.

Con el fin de conocer el impacto de la implementación del Provee, el PEV consideró realizar algunos procesos de investigación en coordinación con el doctor Ricardo Almeida Uranga, líder del Cuerpo de Estudios en Educación y Ciencias Sociales (núm. 54) de la UACJ. A continuación, se mencionan los objetivos, así como hallazgos y conclusiones principales de la investigación de impacto que se presentó en marzo de 2011 ante la comunidad educativa en Ciudad Juárez.

Dicha investigación aborda y da continuidad a los resultados de las investigaciones 2009 y 2010, considerando el análisis longitudinal de datos generados. La pregunta de investigación que orienta el estudio es: ¿En qué medida el Proyecto de Valores Éticos en la Escuela (Provee) que desarrolla el Programa Educación en Valores (PEV) en escuelas del nivel básico en Ciudad Juárez, Chihuahua, contribuye al fortalecimiento del proyecto escolar y, por ende, a la vivencia de una moralidad social establecida desde el colectivo escolar? Para responder a tal cuestionamiento, se diseñó una investigación con enfoque mixto, de dos etapas. Tal como lo sugiere Hernández (2006), el diseño de investigación mixto puede asumir, a la vez, varias modalidades que implican diferentes niveles de complejidad. 
Se precisaron los siguientes objetivos: determinar si las actividades que el colectivo escolar ha planeado en su Provee, han contribuido al mejoramiento de las problemáticas detectadas en el diagnóstico; luego, establecer si existe incidencia del Provee en el clima organizacional y ético de la institución, específicamente en la relación entre maestros-alumnos-padres de familia; y, finalmente, determinar en qué medida los talleres alternativos contribuyen a la construcción de un clima propicio para el desarrollo de un proyecto de valores en las escuelas que los desarrollan.

Se emplearon dos pasos para la captura e interpretación de datos, según el problema de investigación planteado: a) En primera instancia, la captura de datos cuantitativos utilizando el SPSS, en donde se generaron análisis descriptivos y relación de variables; en segunda instancia, se cuantificaron datos cualitativos, los cuales se codificaron y ordenaron en categorías mediante una rejilla de análisis (también conocido como manual de codificación); b) En segundo lugar, se validaron los datos obtenidos en dos procesos anteriores a través de entrevistas a directores, de tal manera que se pudo llegar a una explicación más profunda sobre los factores que se estudiaron.

Se contemplaron dos muestras para el análisis de los datos generados (muestras A y B) en un diseño no experimental longitudinal de tendencia. Es importante señalar que en este tipo de diseños, los sujetos de estudio no son los mismos (pues dentro de las escuelas la muestra en número es idéntica en proporción, pero esta no corresponde a las mismas personas que contestan la encuesta). Sin embargo, la población de referencia es la misma.

La muestra A, considerando los planteamientos de Hernández (2004) en el tratamiento de muestras de orientación hacia la investigación cualitativa, se determinó no probabilística y se contempló a cuarenta y dos escuelas situadas en la fase más avanzada de implementación de su proyecto de valores y que participaron en la evaluación de impacto de 2010, planteles que cumplían su segundo ciclo en el desarrollo de su proyecto de valores (Provee). 
VALORES PARA SER MEJORES: LA INVESTIGACIÓN-ACCIÓN EN UNA PROPUESTA...

Tabla 4. Muestra A. Evaluación de impacto 2011

\begin{tabular}{cccc}
\hline \multirow{2}{*}{$\begin{array}{c}\text { Fechas de levantamiento } \\
\text { de datos }\end{array}$} & \multicolumn{3}{c}{ Núm. de encuestas (muestra A) } \\
\cline { 2 - 4 } & Maestros & Padres & Alumnos \\
\hline Febrero 2009 & 304 & 1540 & 1080 \\
\hline Diciembre 2009 & 304 & 1155 & 957 \\
\hline Diciembre 2010 & 304 & 1153 & 958 \\
\hline Total & 912 & 3848 & 2995 \\
\hline
\end{tabular}

Fuente: Almeida y Ferman, 2011.

Para la muestra B, se consideraron los mismos planteamientos que para la A y se determinó una selección no probabilística de nueve escuelas situadas en la fase $v$ del Provee con nivel de implementación I, que participaron en la evaluación de impacto 2009 y 2010, y que, por tanto, cumplen su tercer ciclo en el desarrollo de su proyecto de valores (Provee), considerándose como las escuelas que deberían presentar mejores resultados en el impacto.

Tabla 5. Muestra B. Evaluación de impacto 2011

\begin{tabular}{cccc}
\hline \multirow{2}{*}{$\begin{array}{c}\text { Fechas de levantamiento } \\
\text { de datos }\end{array}$} & \multicolumn{3}{c}{ Núm. de encuestas (muestra B) } \\
\cline { 2 - 4 } & Maestros & Padres & Alumnos \\
\hline Marzo 2008 & 206 & 642 & 966 \\
\hline Diciembre 2008 & 156 & 896 & 570 \\
\hline Noviembre 2009 & 76 & 522 & 308 \\
\hline Diciembre 2010 & 81 & 302 & 362 \\
\hline Total & 519 & 2362 & 2206 \\
\hline
\end{tabular}

Fuente: Almeida y Ferman, 2011.

En ambos casos el análisis de los datos se realizó con diseño longitudinal, que se fundamenta en hipótesis de diferencia de grupos y correlacionales, con análisis de datos recolectados en dos momentos: entrada y salida. En la tabla 6 se enlistan las dimensiones conceptuales que fueron analizadas en la presente investigación de impacto 2011. 
Tabla 6. Categorías de análisis por grupo. Evaluación de impacto 2011

\begin{tabular}{|c|c|c|}
\hline & Núm. & Categoría \\
\hline \multirow{4}{*}{ Estudiantes } & i & Relaciones interpersonales entre los maestros \\
\hline & ii & Utilización de recursos de apoyo \\
\hline & iii & Relación de los alumnos con los padres \\
\hline & iv & Relación de los padres con la escuela \\
\hline \multirow{4}{*}{$\begin{array}{l}\text { Padres de } \\
\text { familia }\end{array}$} & i & Comunicación y relación de los padres con la escuela \\
\hline & ii & $\begin{array}{c}\text { Participación de los padres en las actividades de la } \\
\text { escuela }\end{array}$ \\
\hline & iii & $\begin{array}{l}\text { Establecimiento de objetivos, actuaciones y valoracio- } \\
\text { nes en la escuela }\end{array}$ \\
\hline & iv & Apoyo al alumno en las tareas escolares \\
\hline \multirow{6}{*}{ Maestros } & i & Comunicación e información internas \\
\hline & ii & Formación y desarrollo profesional \\
\hline & & $\begin{array}{c}\text { Establecimiento de objetivos, actuaciones y valoracio- } \\
\text { nes }\end{array}$ \\
\hline & iv & Progreso profesional y remuneración \\
\hline & & $\begin{array}{c}\text { Conocimiento de los valores, misión y visión de la } \\
\text { escuela }\end{array}$ \\
\hline & vi & Relaciones de trabajo \\
\hline
\end{tabular}

Fuente: Almeida y Ferman, 2011.

Las dimensiones evaluadas fueron generadas en un ejercicio de análisis de diagnósticos realizados en las escuelas por parte del $\mathrm{PEV}$. Las dimensiones e indicadores se relacionan con el ambiente educativo y se parte del supuesto de que su presencia y dinamismo afectan la educación valoral o formación de ciudadanía en los estudiantes. Para el análisis, se generó un conjunto de bases de datos con la información de entrada, correspondiente a febrero de 2009, y de salida, correspondiente a diciembre de 2010, de acuerdo con el rol de los participantes, en hojas de captura del sPSS. Se utilizó la prueba t-Student para determinar la significatividad de las diferencias entre las medias.

A continuación, se detallan las principales diferencias entre las medias en cada una de las categorías y momentos de aplicación. La columna "Dif." señala la diferencia estadística de la media de la respuesta "pre" versus la media de la respuesta "post" en la escala 
indicada. La columna "Sig." indica el nivel de significancia de la prueba t-Student.

Tabla 7. Diferencias y niveles de significancia en las mediciones entre febrero de 2009 y diciembre de 2010 con estudiantes

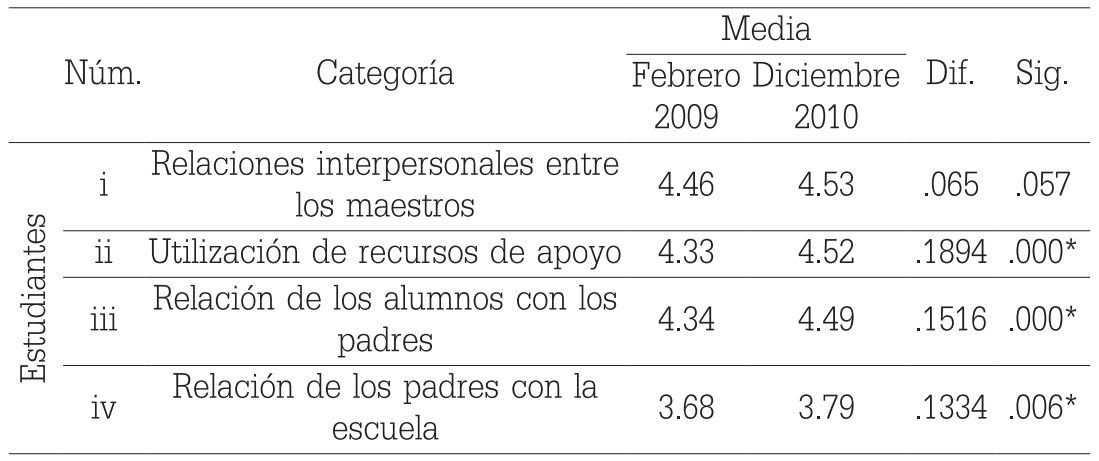

* Son diferencias significativas a un nivel igual a 0.05, es decir, por lo menos 95 $\%$ de confianza.

Fuente: Almeida y Ferman, 2011.

Tabla 8. Diferencias y niveles de significancia con padres de familia. Evaluación de impacto 2011

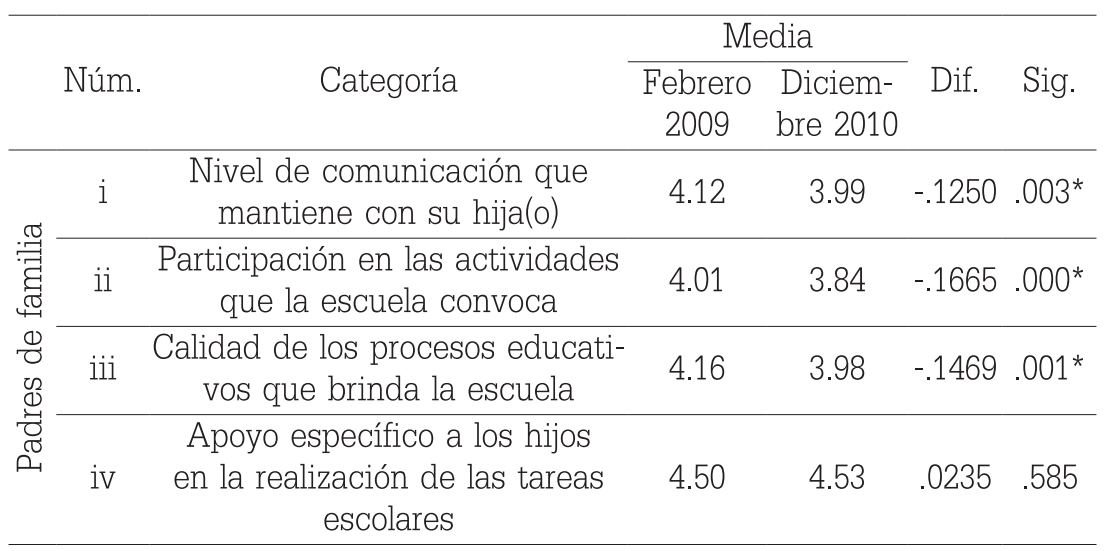

Fuente: Almeida y Ferman, 2011. 


\section{Oliverio Ismael Ferman Ávila}

Tabla 9. Diferencias y niveles de significancia con maestros.

Evaluación de impacto 2011

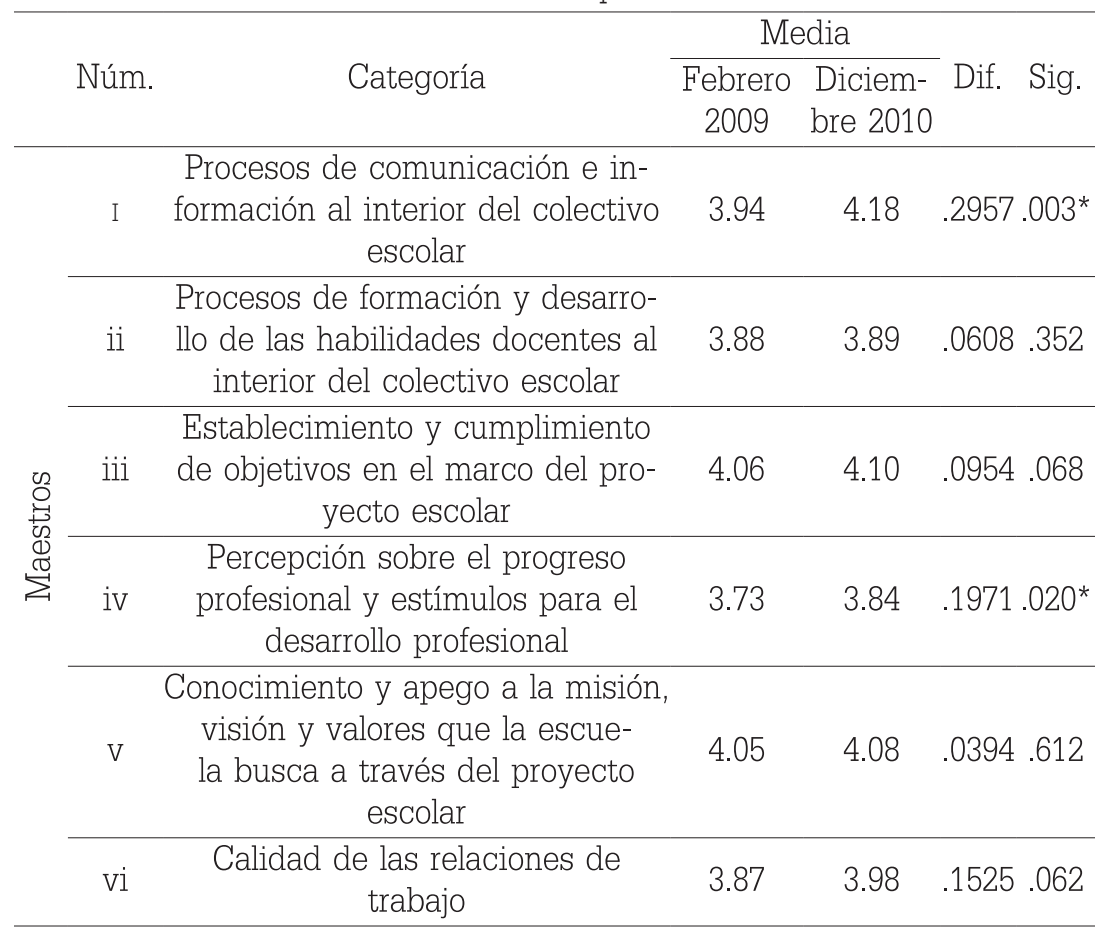

Fuente: Almeida y Ferman, 2011.

Tomando como base los objetivos planteados para la evaluación y los resultados de significancia estadística presentados en las tablas anteriores, la investigación liderada por el doctor Almeida destacó como hallazgos principales los siguientes: un factor muy importante es la utilización de los recursos o materiales didácticos que apoyan la labor docente. Desde la óptica propia de los estudiantes hay una mayor aplicación de dichos materiales en la práctica pedagógica de los docentes; los estudiantes reportan relacionarse más con sus padres. También perciben mayor involucramiento de sus padres en la escuela. Se gana en calidad en las relaciones entre los tres grupos de actores: estudiantes, padres y docentes; mejora la calidad del clima social en la escuela, debido a que hay más relación entre estudiantes con sus padres y de estos 
con la escuela. Si se asume que los "valores" son acuerdos sociales que se construyen en la relación cotidiana entre hijos, padres y docentes, por lo menos desde la perspectiva de los jóvenes sí se ha avanzado en este aspecto de un clima educativo más receptivo y de mayor respaldo al desarrollo social de los estudiantes.

De seis categorías que se evalúan con los docentes, se destaca que solo en dos de ellas existen diferencias significativas. Estas dos categorías corresponden a los procesos de comunicación e información al interior del colectivo escolar; y el factor que tiene que ver con el progreso profesional y los estímulos para el desarrollo profesional. Los otros cuatro factores que se perciben sin cambios son los que tienen que ver con: a) Procesos de formación y desarrollo de las habilidades docentes al interior del colectivo escolar; b) Establecimiento y cumplimiento de objetivos en el marco del proyecto escolar; c) Conocimiento y apego a la misión, visión y valores que la escuela busca a través del proyecto escolar; y d) Calidad de las relaciones de trabajo. Hay que ser muy cautelosos al intentar establecer inferencias sobre estos resultados, ya que se han realizado aproximadamente seis levantamientos de datos con los mismos instrumentos (o una versión ligeramente modificada de ellos). Al considerar los resultados, se puede ver que sí hay una mejora en el clima escolar en los aspectos que tienen que ver con la comunicación y el desarrollo para el profesorado en las escuelas.

Los maestros perciben un mayor flujo en la comunicación y mayores posibilidades de progreso. Se aprovechan los estímulos para el desarrollo. Esto tiene influencia en un mayor arraigo y comunicación entre los grupos. Se gana en calidad en las relaciones; mayor congruencia entre lo que se dice y lo que se hace; mayor congruencia entre lo que se planea y lo que se implementa; mayor congruencia entre lo que se valora y lo que se respeta; mayor entrega y atención educativa por parte de los docentes. Existe mejora en los siguientes aspectos: a) El clima de interacción social; b) Desarrollo de acuerdos sociales, a través de la gestión del currículo; c) Relaciones de mayor solidaridad; d) El Provee orienta en parte las acciones del colectivo de maestros y de los directivos. 
Hasta este punto, el PEv propuso a través de las últimas tres evaluaciones de impacto dos marcos de investigación: la reconstrucción de valores a través de la orientación transversal de la gestión del currículo; y la construcción de valores y desarrollo de actitudes, a través del propio proyecto institucional de gestión escolar mediante una asesoría permanente y sistemática. Por ello, el PEV, con el apoyo técnico y financiero de la asociación civil Fortalessa, ${ }^{5}$ desarrolló una nueva propuesta de investigación que fue llevada a cabo entre junio y julio de 2013. Este estudio tuvo como propósito recabar información acerca de la disposición a la práctica de valores, así como las conductas de riesgo presentes en niños y jóvenes de primarias y secundarias ubicadas en Ciudad Juárez, en las que intervino el PEV durante el ciclo escolar 2012-2013.

Considerando que las escuelas habían implementado un Provee en los cuatro últimos ciclos, se determinó necesario valorar, más allá de los indicadores generales del clima moral, el impacto que su implementación tuvo en los alumnos de las escuelas donde el PEV desarrolló dicha propuesta. Por tanto, esta investigación se centró en valorar la disposición a la práctica de valores de los estudiantes que acuden a planteles que han desarrollado hasta cuatro veces de manera consecutiva un proyecto de valores, considerándose como las escuelas más avanzadas en la implementación del proyecto de valores versus las que se ubican en la fase inicial de la propuesta. La investigación fue a partir de una metodología mixta, un estudio comparativo, con un grupo control y otro experimental. Adicionalmente, se valoró la prevalencia de conductas de riesgo, que se consideran indicadores externos de los contextos escolar, familiar y social inmediatos del estudiante.

Esta investigación se presentó como un estudio exploratorioevaluativo del impacto del proyecto de intervención que el PEv ha desarrollado en escuelas de educación básica en Ciudad Juárez, y pretendió dilucidar algunas características comunes que presentaban los planteles más exitosos en este proceso de intervención. La investigación se determinó desde el enfoque mixto, con un diseño

5 Para conocer acerca del trabajo que desarrolla Fortalessa, puede consultarse su página oficial: http://fortalessa.org.mx/ 
de dos etapas. Tres modalidades se emplearon para la recolección e interpretación de datos, según el problema de investigación planteado: en primera instancia, la cualificación de datos cuantitativos, en este caso en concreto con la ayuda del SPSS, en donde se generaron análisis descriptivos y relación de variables que se convertirán en aspectos cualitativos también sujetos a análisis; en segunda instancia se cuantificaron datos cualitativos, siendo estos codificados y ordenados en categorías de análisis mediante una rejilla de análisis (también conocido como manual de codificación); por último, se validaron los datos obtenidos en dos procesos anteriores, a través de grupos focales y entrevistas a profundidad (Ferman, \& Gallardo, 2013). Con el fin de responder a las preguntas de la investigación, comprobar o desechar la hipótesis, se establecieron cuatro muestras probabilísticas con diferentes diseños, tal como se muestra en la tabla 10.

Tabla 10. Muestras en la evaluación de impacto 2014

\begin{tabular}{|c|c|c|c|c|}
\hline Muestra & Criterio de selección & $\begin{array}{l}\text { Diseño de inves- } \\
\text { tigación - Fase } \\
\text { cuantitativa }\end{array}$ & $\begin{array}{l}\text { Total de } \\
\text { escuelas }\end{array}$ & Muestra \\
\hline \multirow{2}{*}{ A } & $\begin{array}{l}\text { 1. Escuelas que se ubican } \\
\text { la fase v del primer ciclo de } \\
\text { implementación del Provee }\end{array}$ & $\begin{array}{l}\text { Diseño no experi- } \\
\text { mental longitudi- } \\
\text { nal de tendencia } \\
\text { (trend). }\end{array}$ & 261 & 57 \\
\hline & $\begin{array}{l}\text { 2. Escuelas que se ubican en } \\
\text { las fases i-iv del primer ciclo } \\
\text { de implementación del Provee }\end{array}$ & $\begin{array}{l}\text { Diseño no experi- } \\
\text { mental longitudi- } \\
\text { nal de tendencia } \\
\quad \text { (trend) }\end{array}$ & 205 & 206 \\
\hline B & $\begin{array}{l}\text { Escuelas que se ubican en la } \\
\text { fase v del segundo ciclo de } \\
\text { implementación del Provee }\end{array}$ & $\begin{array}{l}\text { Diseño longitudi- } \\
\text { nal de panel }\end{array}$ & 96 & 60 \\
\hline $\mathrm{C}$ & $\begin{array}{l}\text { Escuelas que se ubican en } \\
\text { la fase v del tercer ciclo de } \\
\text { implementación del Provee }\end{array}$ & $\begin{array}{l}\text { Diseño longitudi- } \\
\text { nal de panel }\end{array}$ & 6 & 6 \\
\hline
\end{tabular}

Fuente: Ferman \& Gallardo, 2013. 
De esta evaluación el PEV solo presentó el diseño, pero no las conclusiones y los resultados, tal como puede constatarse en el sitio oficial. ${ }^{6}$

En el ciclo escolar 2013-2014, el PEV puso a consideración de las escuelas la implementación del Modelo de Escuela PEV (MEPEV), una estrategia que buscó hacer más evidente la práctica de valores mediante acciones concretas y cotidianas en el contexto escolar. Este modelo se centra en la práctica de cinco valores centrales: responsabilidad, respeto, honestidad, solidaridad y perseverancia. Asimismo, se busca la construcción de ambientes propicios para el desarrollo de valores y actitudes deseables mediante sinergia entre la escuela y la familia, con el fin de proveer experiencias positivas y la reducción de factores de riesgo (Ferman, \& Gallardo, 2014).

Para la evaluación de los impactos de la implementación del MEPEV, se aplicaron instrumentos en pretest y post-test. En total, se emplearon instrumentos en 364 escuelas en el pretest y 264 en el post-test. La aplicación de los instrumentos del pretest, se llevó a cabo entre octubre y noviembre de 2013, en tanto que el post-test a partir de la segunda semana de junio y hasta la primera de julio de 2014. Para la evaluación de impacto global de la implementación del MEPEV, se consideró para el análisis una muestra de 20 \% (129 escuelas: 3622 instrumentos). Esta evaluación de impacto consideró el desarrollo de valores y actitudes deseables en los estudiantes de los planteles que implementan el MEPEV. El análisis consideró tanto indicadores internos como externos, determinando como factores internos lo referente a la predisposición para la práctica de valores y el desarrollo de actitudes deseables, y como factores externos indicadores del contexto social que pueden ser propicios o no para que el estudiante externe comportamientos deseables (Ferman, \& Gallardo, 2014).

La evaluación consideró tres objetivos: establecer el incremento en la práctica de valores en estudiantes a través del comparativo de resultados entre el pretest y el post-test; instaurar la incidencia de la implementación del MEPEV en la construcción de

6 Reporte incompleto de la evaluación, puede revisarse en: http://www.educacionyvaloresj.org/biblioteca/transparencia/18-evaluacion-de-impacto-2012-2013/file 
ambientes propicios para la práctica de valores en la escuela, con la participación de maestros y padres de familia; y establecer la disminución de factores de riesgo y determinar el nivel de contención contra estos factores como incidencia de la implementación del MEPEV. El diseño de la investigación se efectuó con el enfoque mixto de dos etapas.

Dos modalidades se emplearon para la recolección e interpretación de los datos, según el problema de investigación planteado: en primera instancia, la cualificación de datos cuantitativos, a partir del contraste de los resultados entre el pretest y el post-test. El análisis se llevó a cabo con el spss, en donde se generaron análisis descriptivos y relación de variables que se convirtieron en aspectos cualitativos también sujetos a análisis; en segunda instancia, se analizaron los datos cualitativos generados a través de los grupos focales. Esta información se codificó y organizó de acuerdo con las categorías de análisis determinadas.

Tabla 11. Muestras para pre y post-test

\begin{tabular}{clc}
\hline Muestra & Criterio de selección & $\begin{array}{c}\text { Diseño de investigación - } \\
\text { Fase cuantitativa }\end{array}$ \\
\hline Pretest & $\begin{array}{l}\text { Estudiantes de escuelas } \\
\text { que desarrollan el MEPEV }\end{array}$ & \\
\cline { 1 - 2 } Post-test & $\begin{array}{l}\text { Estudiantes de escuelas } \\
\text { que desarrollan el MEPEV }\end{array}$ & \\
\hline
\end{tabular}

Fuente: Ferman \& Gallardo, 2014.

Para el desarrollo de la evaluación, se determinó una muestra probabilística con un nivel de confianza de 90-95 \% y margen de error entre 0.05-0.10 \%, utilizando el programa estadístico Stats 2.0. A continuación, se establece la muestra tanto para el pretest como el post-test. 


\section{Oliverio Ismael Ferman Ávila}

Tabla 12. Total de estudiantes evaluados según la muestra

\begin{tabular}{ccccc}
\hline $\begin{array}{c}\text { Criterio de } \\
\text { selección }\end{array}$ & Escuelas & Muestra & $\begin{array}{c}\text { Estudiantes } \\
\text { evaluados }\end{array}$ \\
\hline Pretest & $\begin{array}{c}\text { 1. Escuelas que desarrollan el } \\
\text { MEPEV. }\end{array}$ & & & \\
& $\begin{array}{c}\text { Aplicación en octubre-noviem- } \\
\text { bre de 2013 }\end{array}$ & 364 & 72 & 2160 \\
\hline \multirow{2}{*}{$\begin{array}{c}\text { 2. Escuelas que desarrollan el } \\
\text { MEPEV. }\end{array}$} & 264 & 53 & 1590 \\
& $\begin{array}{c}\text { Aplicación en junio-julio de 2014 } \\
\text { Total }\end{array}$ & 314 & 125 & 3750 \\
\hline
\end{tabular}

Fuente: Ferman \& Gallardo, 2014.

De los resultados obtenidos en la evaluación de impacto, en este artículo se destacan específicamente los que refieren al desarrollo de actitudes de los estudiantes. En la tabla 13, se muestran los resultados de siete indicadores en esta dimensión.

Tabla 13. Comparativo de actitudes de los estudiantes

\begin{tabular}{cccc}
\hline Indicador & $\begin{array}{c}\text { Resultado } \\
\text { pretest } \\
(\%)\end{array}$ & $\begin{array}{c}\text { Resultado } \\
\text { post-test } \\
(\%)\end{array}$ & $\begin{array}{c}\text { Dif. } \\
(\%)\end{array}$ \\
\hline Motivación por sus logros & 93 & 93.59 & 0.59 \\
\hline Desarrollo de la capacidad para decir no & 82.6 & 82 & -0.6 \\
\hline Solución pacífica de conflictos & 61.3 & 62.88 & 1.58 \\
\hline Desarrollo de una visión de futuro & 80.8 & 84 & 3.2 \\
\hline Desarrollo del control de impulsos & 68.6 & 70.13 & 1.53 \\
\hline $\begin{array}{c}\text { Capacidad para sobreponerse a situaciones } \\
\text { adversas }\end{array}$ & 80.6 & 76.12 & -4.48 \\
\hline Comportamientos violentos & 21.9 & 20 & -1.9 \\
\hline
\end{tabular}

Fuente: Ferman \& Gallardo, 2014.

En la tabla de resultados pueden destacarse los siguientes resultados, a partir del trabajo desarrollado por las escuelas durante el desarrollo del MEPEV: 1.58 \% de las niñas y niños desarrollaron capacidades para resolver conflictos a través del diálogo, esto es, 2844 niñas y niños que son menos violentos. Este resultado se 
relaciona directamente con lo comentado por los directores de las escuelas que implementaron el MEPEV. El $55 \%$ aduce que los niños mejoraron en la práctica del respeto, mientras que $14 \%$ menciona la disminución de peleas y violencia como un efecto de la implementación del MEPEV; a pesar de los resultados externados en el punto 1, 1.9 \% más de los niños tuvo algún comportamiento violento; las actividades que desarrollaron las escuelas con la implementación del MEPEV lograron que $3.2 \%$ más de los niños desarrollaran una visión de futuro, esto es, un impacto positivo en 3700 niñas y niños; un aspecto que debe ser considerado como alarmante, tanto por las escuelas, nuestro programa y las autoridades educativas, es que entre noviembre (pretest) y julio (post-test) $4.48 \%$ de niñas y niños menos pueden sobreponerse a situaciones adversas. Esta cifra representa alrededor de 8000 niños que resienten los efectos del contexto inmediato.

Tabla 14. Resultados de la práctica de valores

\begin{tabular}{cccc}
\hline Indicador & $\begin{array}{c}\text { Resultado } \\
\text { pretest } \\
(\%)\end{array}$ & $\begin{array}{c}\text { Resultado } \\
\text { post-test } \\
(\%)\end{array}$ & $\begin{array}{c}\text { Dif. } \\
(\%)\end{array}$ \\
\hline Uso de alcohol & 23.46 & 12.76 & -10.7 \\
\hline Uso de tabaco & 5.4 & 4.55 & -0.85 \\
\hline Uso de drogas & 6.2 & 2.5 & -3.7 \\
\hline Conductas violentas y/o antisociales & 17.8 & 12.4 & -5.4 \\
\hline Tendencia a la depresión & 28.7 & 35.58 & +6.88 \\
\hline Tendencia al suicidio & 20.4 & 9.8 & -10.6 \\
\hline
\end{tabular}

Fuente: Ferman \& Gallardo, 2014.

No puede considerarse un efecto directo de la implementación del MEPEV, pero sí un efecto colateral resultado del trabajo conjunto del PEv, la escuela — que desarrolla las estrategias planteadas- y la familia. Siempre habrá mayores posibilidades de práctica de valores en un ambiente propicio, donde se reducen factores de riesgo que se consideran inhibidores de la práctica de valores y actitudes deseables para el desarrollo de los estudiantes en una sociedad. 
De la tabla 14 se destacan los siguientes resultados, a partir del trabajo desarrollado por las escuelas durante la exposición del MEPEV: 10.7 \% de niñas y niños dejaron de consumir alcohol entre noviembre de 2013 y julio de 2014, para situar la presencia del factor de riesgo en $12.76 \%$ de los niños evaluados. El impacto se establece en casi 20000 niñas y niños, que es un resultado que puede considerarse alentador; sin embargo, todavía casi 23000 niñas y niños están expuestos a este vicio socialmente aceptado, por lo que la escuela debe seguir asumiendo la tarea de educar a los padres si se quiere que el impacto sea mayor; el uso del tabaco disminuyó en 0.85 \% (1500 niños menos) en tanto que el uso de drogas $3.7 \%$, esto es, 6600 niñas y niños. Aun con la disminución, debe seguirse trabajando en el área de prevención ligada con la práctica de valores mediante la práctica de valores; en este apartado, las conductas antisociales (pintas de la escuela, grafiti, participación en pandillas, bandas, entre otras) disminuyeron en 5.4 $\%$, cifra que representa a casi 10000 niñas y niños que dejaron de hacerlo con respecto a la evaluación inicial (pretest); un factor de mucho riesgo que debe ser atendido a través de un plan intersectorial, es la tendencia a la depresión, factor que aumentó en 6.88 \%, esto es, 12300 niñas y niños más que se deprimen a menudo.

Otros resultados que dan muestra del avance de las escuelas que implementaron el MEPEV son: en general, los directores observaron que 13 \% más de los estudiantes tiene una mayor práctica de valores; mejoró la actitud y trabajo colaborativo entre los docentes en $13 \%$; la limpieza y orden mejoró sustancialmente en 11 $\%$ de las escuelas en el primer año de la campaña permanente de orden y limpieza; 12 \% de las escuelas refirió un aumento en la participación de los padres mediante las actividades que se planearon en conjunto con el PEV. La mejora referida fue un aumento en la participación en $44 \%$ con respecto a la del inicio del ciclo escolar. Sin embargo, el involucramiento de los padres continuó como foco rojo, ya que se detectaron escuelas cuyo porcentaje de participación y vinculación con los padres no rebasa el $5 \%$ de la población total de alumnos; 82 \% de las escuelas mejoró en el cumplimiento del proyecto escolar; 72 \% de las escuelas trabajó 
con el reglamento escolar y la normatividad, aspecto que tiene efecto directo en la asunción de la responsabilidad de las tareas por parte de los docentes y directivos; 64 \% de las escuelas pudieron vincular el Consejo Escolar de Participación Social (CEPS) de mejor manera. En la mayoría de los casos fue el CEPS quien formó parte del Comité de Valores que desarrolló las actividades del MEPEV; y 36 \% de las escuelas que no tenía una misión, visión y valores visibles hacia la comunidad al inicio del ciclo escolar, fue colocado como resultado de la evaluación inicial del MEPEV.

Entre 2015 y 2017 el PEV continuó desarrollando el Modelo de Escuela con algunas modificaciones orientadas a fortalecer la práctica de la cultura de la legalidad en los planteles, proyecto que fue subvencionado por el Departamento de Estado de Estados Unidos, a través de la Iniciativa Mérida. Con el propósito de valorar los resultados de esta intervención, la dirección ejecutiva del PEV decide realizar un estudio evaluativo que fue dirigido por el Cuerpo Académico 51 de Psicología Aplicada de la UACJ, el cual tuvo como coordinador al doctor Jesús Humberto Burciaga y como responsable técnica a la maestra Verónica Portillo Pérez. Esta evaluación correspondió a la intervención del PEV en la escuela durante el ciclo escolar 2015-2016, cuyos resultados se presentaron ante la comunidad educativa en Ciudad Juárez el 7 de noviembre de 2017.

El estudio fue de corte descriptivo y comparativo, y utilizó perspectivas e instrumentos, tanto cualitativos como cuantitativos. Los objetivos que persiguió la evaluación fueron: comparar estadísticamente las puntuaciones de las escuelas participantes antes y después de la primera fase de implementación del proyecto en la variable de prevención de conductas de riesgo; comparar estadísticamente las puntuaciones de los planteles participantes antes y después de la primera fase de implementación del proyecto en la variable de cultura de la legalidad; comparar estadísticamente las puntuaciones de las escuelas participantes antes y después de la primera fase de implementación del proyecto en la variable de convivencia sana; así como conocer la experiencia de profesores, profesores enlace y directores de planteles participantes en el programa en relación con los elementos que favorecen y dificultan su 
implementación. Para realizar la comparación estadística, se incluyeron datos de la totalidad de las escuelas que participan en el proyecto que implementó el PEv: en total 493 planteles. En tanto que para la fase cualitativa, se llevó a cabo un muestreo intencional de 12 profesores, 17 directores y 6 profesores enlace de las escuelas participantes para integrar los grupos focales (Burciaga, 2017).

Los datos cuantitativos se obtuvieron mediante la codificación de los registros iniciales y después de la primera fase del programa en relación con las variables establecidas (prevención de conductas de riesgo, cultura de la legalidad y convivencia). Estos registros se integraron a partir de los cuestionarios sobre indicadores de logro para cada variable, instrumentos que fueron diseñados por el propio programa de valores.

De acuerdo con Burciaga (2016), en el análisis de datos se compararon datos mediante una prueba t-Student, con pre y posttest, utilizando el paquete estadístico PSPP 0.10.2. El análisis cualitativo se efectuó a través de un análisis matricial con categorías preestablecidas (factores que favorecen y factores que dificultan la implementación de la propuesta PEV) de las transcripciones de los cuatro grupos focales utilizando el paquete HyperResearch 3.7.

En cuanto a los resultados más significativos, se muestra que la variable de cultura de la legalidad mostró un puntaje de 35.19 en promedio antes de iniciar el proyecto en las escuelas participantes. Al concluir la primera fase de implementación, los mismos planteles calificaron con 48.15 puntos en promedio, lo cual significa que las escuelas participantes se elevaron en 12.96 puntos en promedio. Esta diferencia resultó estadísticamente significativa al comparar las dos aplicaciones del mismo grupo mediante una prueba t-Student (Burciaga, 2017). 
VALORES PARA SER MEJORES: LA INVESTIGACIÓN-ACCIÓN EN UNA PROPUESTA...

Tabla 15. Cultura de la legalidad

\begin{tabular}{ccccc}
\hline Variable & Promedio & $\begin{array}{c}\text { Diferencia de } \\
\text { medias }\end{array}$ & $\begin{array}{c}\text { Significancia } \\
\text { estadística }\end{array}$ \\
\cline { 1 - 2 } Cultura de la legalidad antes & 35.19 & & 12.96 & .000 \\
\cline { 1 - 2 } Cultura de la legalidad después & 48.15 & & \\
\hline
\end{tabular}

Fuente: Burciaga (2017).

En lo que respecta a la prevención de conductas de riesgo obtuvo un puntaje inicial de 21.53 puntos en promedio. Después de la implementación de la primera etapa del proyecto el promedio fue de 34.71 puntos, lo que representa una diferencia estadísticamente significativa de 13.18 (Burciaga, 2017). El indicador de convivencia sana registró un valor antes de la implementación de la primera etapa del proyecto de 28.65 puntos y al finalizar obtuvo una calificación promedio de 38.72 .

Los resultados del estudio permiten, por un lado, afirmar que la implementación del proyecto del PEV tuvo un impacto favorable de, al menos, diez puntos en las variables de prevención de conductas de riesgo, cultura de la legalidad y convivencia sana. Como principales fortalezas detectadas destacan: la aceptación del proyecto por parte de los usuarios; el reconocimiento de actividades con gran impacto en los planteles; la comunicación efectiva del asesor con las escuelas; el involucramiento de las autoridades de los planteles en el proyecto; la posibilidad de adecuar el proyecto a las necesidades particulares de cada escuela; las herramientas y materiales que la participación en el proyecto brinda; los logros académicos en los planteles relacionados con el éxito del proyecto; la integración y complementariedad del proyecto con la ruta de mejora de las escuelas; y el involucramiento y compromiso de los docentes en los planteles (Burciaga, 2017).

\section{CONCLUSIONES}

En la actualidad la situación social obliga a debatir el impacto de la educación en el desarrollo de actitudes y valores deseables en los estudiantes, particularmente en lo que se refiere a la edu- 
cación básica. El estado del conocimiento sobre una propuesta de educación en valores presentado mediante las investigaciones antes expuestas, refleja en el PEV una constante preocupación y ocupación por determinar potencialidades y debilidades, con el firme propósito de fortalecer su proceso de intervención en las escuelas de educación básica en Ciudad Juárez, Chihuahua. Esta ocupación se refleja en una constante búsqueda de condiciones o variables que le ayuden a obtener mejores resultados para quienes implementen su propuesta.

Cada una de las experiencias de investigación antes abordadas exponen variables y/o condicionantes, pero no han logrado precisar en dónde se ubica el punto de quiebre entre la teoría (la propuesta del PEv) y la práctica que desarrollan los maestros en el contexto escolar. A partir de indagaciones sobre algunos aspectos que pudieran afectar directamente a esta y a cualquier otra propuesta de formación en valores que se dirija a los docentes, se encontró que un acercamiento a los campos ideológicos en los maestros que desarrollan la propuesta pudiera dar luz a la constante búsqueda que, hasta ahora, ha caracterizado al PEV.

Los resultados presentados cobran mayor relevancia por ser una propuesta gestada en una organización de la sociedad civil y operada en el sistema de educación público en Ciudad Juárez. Aunado a esto, su intervención continúa avalada por un convenio de colaboración formal entre la Secretaría de Educación y Deporte del Gobierno del Estado de Chihuahua, pero particularmente porque las investigaciones se han desarrollado con diferentes agentes: dependencias gubernamentales, universidades públicas, universidades privadas, académicos e investigadores independientes. Aspecto en particular que muestra la visión del PEV de estrechar vínculos - que le posibiliten conocer los impactos de su intervención socioeducativa - con instituciones y personas interesadas en el desarrollo de investigaciones y/o proyectos en el ámbito educativo, y particularmente en el campo de la educación en valores.

Actualmente, el PEV desarrolla lo que denomina MEPEV con la orientación de fortalecer el desarrollo de una cultura de la legalidad en el contexto escolar. El MEPEV es una estrategia educativa 
de intervención socioeducativa que el PEV desarrolla en, por lo menos, quinientas escuelas de educación básica en Ciudad Juárez durante el ciclo escolar 2017-2018. El PEV lo denomina modelo, porque en la estrategia de intervención se consideran indicadores y metas de logro evidentes que permiten observar la práctica de valores en las actividades cotidianas de la escuela. En el MEPEV se considera el desarrollo formativo mediante la práctica cotidiana de cinco valores que son el eje de toda acción en el mismo modelo: responsabilidad, respeto, honestidad, perseverancia y solidaridad. El MEPEV se lleva a cabo mediante una serie de actividades que se organizan en cinco pasos, que tienen su inicio y final con el ciclo escolar, que incluyen tanto los diagnósticos y el plan de trabajo como la puesta en práctica de las actividades formativas y la medición de los resultados.

Desde el ciclo escolar 2015-2016 y hasta el 2017-2018, la propuesta del PEV fue financiada, en gran medida, por el Bureau of International Narcotics and Law Enforcement Affairs del Departamento de Estado de Estados Unidos dentro del Programa de Fortalecimiento de Cultura de la Legalidad. Actualmente, el proyecto padece una crisis severa de financiamiento que dificulta la implementación de esta propuesta valoral que durante veinticuatro años ha permeado en la cultura escolar y ha evidenciado logros importantes. El impacto del Programa Educación en Valores, A. C., debe ser medido, desde nuestra consideración, a contrario sensu, es decir, poder definir qué hubiera sucedido sin el apoyo y la presencia del PEV en las escuelas es muy difícil: autoridades educativas, maestros, padres de familia, pero sobre todo los miles de estudiantes que han pasado por las aulas de los planteles que han implementado el PEV durante estos veinticuatro años de existencia en la comunidad juarense hacen evidente la naturaleza del propio programa, y permiten que, de continuar obteniendo impactos - a corto, mediano y largo plazos - en el desarrollo de actitudes deseables, se fortalezca el anhelo de que los valores nos ayuden a ser mejores como sociedad. 


\section{REFERENCIAS}

Almeida, R. \& Ferman, O. I. (2011). Evaluación de impacto en escuelas que desarrollan el Proyecto de Valores Éticos (PROVEE) con el mayor nivel de implementación en primer y segundo ciclos de mejora continua. Un estudio longitudinal. Programa Educación en Valores A.C. y Universidad Autónoma de Ciudad Juárez. Cuerpo Académico de Educación y Ciencias Sociales. Consulta realizada el 13 de Febrero de 2018, en http://www. educacionyvaloresj.org/biblioteca/transparencia/16-evaluacionde-impacto-2010-2011/file

Anaya, R. (2007). Razonamiento Moral, Ideología Educativa y Programas de Valores. Programa Educación en Valores A.C. y Universidad Autónoma de Ciudad Juárez. Documento inédito. Anaya, R., \& Ferman, O. I. (2012). Evaluación del impacto del proyecto de intervención del Programa Educación en Valores, A. C. en escuelas de educación básica en Ciudad Juárez. Proyecto 2011. Programa Educación en Valores, A. C./Universidad Autónoma de Ciudad Juárez. Recuperado el 12 de febrero de 2018, de http://www.educacionyvaloresj.org/biblioteca/ transparencia/17-evaluacion-de-impacto-2011-2012/file

Arroyo Picard, I. (1998). Filosofía de la educación en Chihuahua. Chihuahua, México: Solar Colecciones/Instituto Chihuahuense de la Cultura/Fondo Estatal para la Cultura y las Artes.

Bismark (2004). Valores: evaluación integral del Programa Educación y Valores. Reporte técnico. Bismark: Buró de Investigación Social \& Marketing. Documento inédito.

Burciaga, J. (Coord.) (2017). Evaluación del proyecto "Valores para fortalecer la cultura de la legalidad” del Programa Educación en Valores, A. C. Informe técnico. Cuerpo Académico 51: Psicología Aplicada de la UACJ. Recuperado el 21 de febrero de 2018, de http://www.educacionyvaloresj.org/transparencia/5informe-tecnico-de-la-evaluacion-del-mepev-2016/file

Buxarrais, M. R. et al. (1999). La educación moral en primaria y en secundaria. Una experiencia española. Biblioteca para la Actualización del Maestro (pp. 60-112). México: oEi/Unesco/SEP. 
----- (1997). La formación del profesorado en educación en valores. Propuesta y materiales (pp. 52-87). Bilbao, España: Desclée De Brouwer.

Coordinación de Investigación y Desarrollo Académico (1998). Evaluación del Programa Integral de Valores: resultados y recomendaciones. Gobierno del Estado de Chihuahua-Dirección General de Educación y Cultura. Documento inédito.

Ferman, O. (2007). La enseñanza de Valores en Correspondencia con la Ideología Educativa del Maestro de Primaria: Una Experiencia en la Frontera Norte de México-Edición Única. Instituto Tecnológico y de Estudios Superiores de Monterrey. Tesis de Maestría.

Ferman, O. I., \& Almeida, R. (2009, 2 de marzo). Evaluación de impacto en escuelas que desarrollan el Proyecto de Valores Éticos (Provee) con el mayor nivel de implementación en el Primer Ciclo de Mejora Continua. Resumen ejecutivo. Programa Educación en Valores, A. C./Universidad Autónoma de Ciudad Juárez-Instituto de Ciencias Sociales y Administración. Recuperado el 28 de enero de 2018, de http://www.educacionyvaloresj.org/biblioteca/transparencia/14-evaluacion-deimpacto-2007-2008/file

Ferman, O. I., \& Gallardo, A. (Coords.) (2013). Evaluación de impacto de su proyecto de intervención en escuelas de educación básica en Ciudad Juárez. Proyecto 2012-2013. Programa Educación en Valores, A. C./Fortalessa. Recuperado el 17 de febrero de 2018, de http://www.educacionyvaloresj.org/biblioteca/transparencia/18-evaluacion-de-impacto-2012-2013/file (2014). Evaluación de impacto de la intervención del Modelo de Escuela PEV (MEPEV) en escuelas de educación básica en Ciudad Juárez. Programa Educación en Valores, A. C./Fortalessa. Recuperado el 17 de febrero de 2018, de http://www. educacionyvaloresj.org/transparencia/19-evaluacion-de-impacto-2013-2014/file

Ferman, O. I., \& Junco, R. I. (2006). Modelo Educativo del Programa Educación en Valores. Chihuahua, México: Talleres Gráficos de Gobierno del Estado de Chihuahua. 
Hernández Sampieri, R., Fernández Collado, C., \& Baptista Lucio, P. (2006). Metodología de la investigación (4a. ed.). México: McGraw-Hill.

Latapí, P. (2003). El debate sobre los valores en la escuela mexicana. México: Fondo de Cultura Económica.

O'Neill, W. (1981). Educational Ideologies: Contemporary Expressions of Educational Philosophy. University of Southern, California. California, USA: Goodyear Publishing Company Inc.

Osio, M. A., Pérez, B. A., Montoya, R. R., Navarro, H. J., \& Herrera, R. A. (1999). Evaluación del Programa de Educación en Valores Progreso. Universidad Autónoma de Ciudad JuárezInstituto de Ciencias Sociales y Administración-Departamento de Ciencias Sociales-Programa de Trabajo Social. Documento inédito.

Pérez, B. A., \& Puga, A. D. (2001). II Evaluación del Programa Educación en Valores. Universidad Autónoma de Ciudad JuárezInstituto de Ciencias Sociales y Administración-Departamento de Ciencias Sociales-Programa de Trabajo Social. Documento inédito.

Pliego, C. (2007). Proyecto Eti-K. Programa EAVE. Recuperado el 14 de enero de 2007, de http://www.etik.com.mx/inicio.php

Suárez Toriello, E. (2005). Evaluación de impacto actitudinal sobre el Programa de Educación en Valores. Reporte de investigación. Recuperado el 30 de enero de 2018, de http://www. educacionyvaloresj.org/biblioteca/transparencia/13-evaluacionde-impacto-2005-2006/file 